\title{
ACEPTACIÓN DE LA VIOLENCIA DE GÉNERO EN DOCENTES DE ESCUELAS PÚBLICAS
}

\section{Acceptance of Gender-Based Violence by Public School Teachers}

Aída Mencía Ripley*

a.mencia@unibe.edu.do

\begin{abstract}
${ }^{1}$ Universidad Iberoamericana, República Dominicana
\end{abstract}

Fecha recepción: 23/08/2019 Fecha aprobación: 09/12/2019

\section{Resumen}

La violencia de género (VG) es una problemática de los sistemas educativos y de salud de la región Latinoamericana. República Dominicana enfrenta altas cifras de VG en sus diversas manifestaciones. Por esta razón, este estudio exploró cómo la aceptación de tres dimensiones de la VG impacta las creencias de docentes de primaria con relación a las habilidades de niñas y niños en distintas asignaturas escolares. Las asignaturas escolares fueron Lectura y Escritura, Matemáticas, Ciencias y Deportes. En República Dominicana la respuesta ante la VG suele ser asistencial y reactiva a un suceso. Existe poca evidencia local sobre cogniciones y barreras actitudinales hacia la prevención de la violencia que permitan identificar cambios y acciones preventivas en la escuela. A través de un estudio con un diseño prospectivo, transversal y correlacional se evaluó el impacto de la aceptación de mitos de la VG en el ámbito escolar. Encontramos que los docentes por lo general aceptan mitos sobre las distintas formas de violencia de género, generalmente culpabilizando a las mujeres por su victimización. De igual manera aceptan estereotipos sobre habilidades académicas en función del género femenino o masculino, especialmente para las matemáticas, para la cual el modelo utilizado explicó un 12\% de la varianza. Este estudio señala que el entorno educativo no solo replica desigualdades sociales, sino que, además, estos sesgos impactan las valoraciones que reciben los estudiantes por parte de sus docentes.

Palabras clave: docentes, escuelas públicas, violencia de género.

\section{Abstract}

Gender-Based Violence (GBV) is a problem in education and health systems across Latin America. The Dominican Republic consistently shows high rates across several types of GBV. Due to the aforementioned reasons, this study explores how the acceptance of three types of GBV impacts the beliefs of primary school teachers regarding the abilities of girls and boys in different academic disciplines. The subjects are Reading and Writing, Mathematics, Science, and Physical Education. In the Dominican Republic, the response to GBV is typically reactive to specific events of victimization. There is little local data on the cognitions and attitudinal barriers to violence prevention that allow scientists to identify change and preventive schoolbased programs. Through a prospective, cross-sectional, correlational study design, we found that teachers generally accept myths or stereotypes about GBV generally blaming women for their victimization. Similarly, they accept stereotypes about academic skills based on the female or male gender, especially for Mathematics, for which the model explained $12 \%$ of the variance. This study indicates that the educational environment not only replicates social inequalities, but that these biases also impact the assessments students receive form their teachers.

Keywords: teachers, public schools, gender-based violence. 


\section{Introducción}

La violencia de género (VG) es una problemática global (Heise, Ellsberg \& Gottmoeller, 2002). Solo en los Estados Unidos de América, se estima que una de cada cinco mujeres será violada en algún momento de su vida (National Sexual Violence Resource Center [NSVRC], 2015). Los contextos educativos no han podido ofrecer protecciones a las mujeres por encima de la vida cotidiana y hasta un $90 \%$ de mujeres que son violadas en el campo universitario no reportan el incidente a las autoridades (NSVRC, 2015). La República Dominicana, al igual que muchos otros países de América Latina, tiene conocidos problemas relacionados con la VG (Barredo et al., 2014). Esta violencia tiene diversas manifestaciones que se expresan desde la violencia obstétrica hasta la violencia que ocurre entre parejas íntimas. Cifras recientes establecen que aproximadamente el $20 \%$ de las dominicanas de más de 15 años de edad han experimentado por lo menos un episodio de violencia sexual, y un $37 \%$ de las nińas que viven en zonas rurales son casadas antes de los 18 años de edad (Plan Internacional, 2017; Sánchez, 2018). Se ańade a esta problemática la diversidad de los sistemas locales para reportar y monitorear los casos de VG y el sub-reporte de casos de violencia machista en diferentes escenarios nacionales.

En muchos casos, las manifestaciones de violencia machista se han normalizado, por lo que los actos violentos no se consideran como tal y continúan manifestándose en contextos familiares. La naturaleza privada o familiar de muchas de las violencias de género (p. ej., violencia entre parejas íntimas, matrimonio infantil informal) impiden que las violencias se visibilicen y que las instancias competentes del Estado actúen para proteger los derechos de las mujeres y, en especial, los derechos de las niñas. Adicionalmente, las mujeres y niñas dominicanas están expuestas a un sinnúmero de tipos de violencia pública, que aún son excusadas como "parte de la cultura", como, por ejemplo, el acoso callejero, la cosificación en el entorno laboral, así como las violencias interseccionales que experimentan las mujeres afrodescendientes y LGBTQ en entornos laborales (Mingo, 2010).
La escuela es el espacio idóneo para la transmisión de valores culturales. La escuela tiene la capacidad de construir conocimientos y fomentar competencias en los estudiantes. A la vez, la estructura organizacional y las relaciones interpersonales que ocurren dentro del contexto escolar tienen el potencial de replicar las desigualdades y violencias que experimentan los múltiples grupos vulnerabilizados en la cultura general. El docente como transmisor principal de valores y constructos culturales puede transmitir prejuicios y estereotipos de manera explícita o implícita mientras se desempeña en su labor docente. En el contexto escolar se ha evidenciado el Efecto Pigmalión, en el cual las expectativas de los docentes tienen una relación directa con el desempeño escolar de los estudiantes (Friedrich et al., 2015). Dadas estas características, las actitudes y creencias de los y las docentes con relación a las violencias de género son fundamentales para la implementación de la educación no sexista y basada en igualdad de derechos. Creencias cónsonas con el sexismo constituyen importantes barreras actitudinales en la implementación de sistemas educativos más justos para todos los estudiantes y para todos los que laboran en entornos académicos, desde la escuela hasta la universidad (Eagly \& Miller, 2016).

\section{Revisión de la literatura}

Ante los retos que suponen las altas cifras de violencia de género, el gobierno dominicano, la sociedad civil y la cooperación internacional han implementado diversos programas de atención a mujeres victimizadas. Se han creado también instancias gubernamentales dedicadas exclusivamente a la atención de la violencia de género y a la promoción de la igualdad de género como mecanismo de prevención de la violencia machista. De igual manera, se han fortalecido los sistemas de protección para niños y niñas victimizados por la violencia intrafamiliar. A pesar de estas importantes iniciativas, la violencia de género - especialmente los feminicidios - persiste en la sociedad dominicana. Si bien existen estas iniciativas, al igual que una creciente conciencia en la sociedad civil sobre la violencia de género, el país 
carece de investigaciones rigurosas y sistematizadas que den explicación a la violencia de género y exploren los mecanismos cognitivos y conductuales que expliquen las barreras a los cambios que promueven las diferentes políticas públicas. Esto deja a la comunidad académica nacional y regional, así como a los creadores de políticas públicas, con poca información, ésta no va más allá de reportes de prevalencia e incidencia de casos (Mingo, 2010).

Para poder disminuir de manera sustancial la violencia de género se necesitan programas preventivos basados en buena evidencia local, así como un sistema legal robusto que permita definir, monitorear y sancionar la violencia de género de manera eficaz. No obstante, estas acciones quedarán incompletas mientras no se exploren los mecanismos a través de los cuales los diferentes sistemas sociales enseñan y refuerzan la violencia y las jerarquías de poder que mantienen la desigualdad. Es en este contexto que la escuela cobra un valor esencial en el discurso de las nuevas feminidades y masculinidades.

En la escuela, los niños y niñas reciben instrucción implícita y explícita sobre asignaturas escolares y sobre valores, ideales y comportamientos valorados en la cultura. De igual manera, la escuela transmite normas claras e implícitas sobre comportamientos y expresiones que la cultura no considera aceptables. La escuela introduce dos problemas importantes en la socialización del sexismo: el sexismo institucional que se transmite a través del poder y la autoridad y el sexismo implícito que se ve reforzado en las relaciones interpersonales que se dan entre docentes, entre alumnos y las diversas figuras de autoridad que se encuentran en el entorno escolar. El entorno educativo actúa como imitador de las estructuras sociales desiguales de su sociedad (López Ojeda, 2006). El docente, como figura principal en el proceso de aprendizaje del niño o niña, podría reforzar el sexismo de estas dos maneras por diversas razones.

En primer lugar, los docentes provienen de la misma cultura sexista que los estudiantes, por lo que su proceso de consolidación de identidad ha ocurrido en un contexto sexista en el cual han interioriza- do los procesos de enseñanza de valores culturales y jerarquías sociales que relegan a la mujer a un rol subordinado (Artal, 2009; Pérez \& Hernández, 2009). En este momento no tenemos indicaciones o evidencia local de que en la formación del docente se atienden temas sobre cómo los docentes han interiorizado guiones culturales sexistas. Hay evidencias también que señalan que las competencias relacionadas con las Ciencias y Matemáticas, se vinculan a las características del liderazgo masculino, mientras que las características femeninas no se correlacionan con competencias propias de las ciencias (Carli et al., 2016).

En segundo lugar, la profesión de la enseñanza o el magisterio, especialmente por estar altamente ocupado por mujeres, trae consigo problemas tangibles relacionados con el sexismo. La transición de la mujer, desde lo que Barquín y Melero (1994) llaman el "espacio íntimo del hogar” al trabajo, sucedió relegando a la mujer a ocupaciones públicas prácticamente iguales a las desempeñadas por la mujer en el hogar (por ejemplo, aquellas profesiones que se enfocan en el cuidado y enseńanza de nińos y nińas).

El ingreso de la mujer de clase media a la vida laboral en profesiones como el magisterio, la enfermería y otras labores de cuidadoras o asistentes, especialmente aquellas vinculadas al cuidado de la niñez, realmente no representan una ruptura de los roles tradicionales y limitados asignados a las mujeres. La brecha salarial a la vez impidió que estas profesiones representaran un verdadero cambio en la jerarquía de poder y la posición de la mujer en tal jerarquía. Esta similitud del rol profesional con el rol del hogar puede también explicar la razón por la cual dichas profesiones siguen eminentemente feminizadas y la remuneración salarial inferior a profesiones masculinizadas. Es por esto que la presencia de la mujer en el aula, en calidad de maestra, representa en sí la personificación de estereotipos culturales sobre el rol de la mujer como aquella cuyo único aporte social yace en la formación o cuidado de la próxima generación. Esto no sugiere que la mujer debe ausentarse del magisterio, sino que esta figura requiere de una mirada crítica y un accionar explícito por parte del 
sistema educativo para poder transformar este rol en uno que permita fomentar la igualdad.

Lo antes expuesto explica parcialmente cómo, a pesar de la participación de las niñas en la educación, no se han visto mayores avances hacia sociedades menos desiguales. Si bien las niñas dominicanas están recibiendo educación pública y privada, a las mismas se les transmiten expectativas sexistas sobre sus capacidades y roles aceptables, por lo que la mera exposición a procesos educativos no es un garante de la equidad. Diversos autores han evidenciado que los docentes tienen expectativas académicas muy marcadas sobre sus alumnos dependiendo del sexo biológico de éstos (Artal Rodríguez, 2009; Barquín \& Melero, 1994). Este es el caso especial de las matemáticas, en las cuales las actitudes y creencias de docentes y alumnos suelen ser mejores indicadores de logro académico (Sánchez-Vincitore et al., 2017). También evidencian que los docentes prestan más atención a los niños varones en el aula, y que desconocen sus propias actitudes sexistas, teniendo poca consciencia de cuándo y cómo transmiten estereotipos de género a sus alumnos. De igual manera, las docentes han expresado que, al igual que las demás mujeres de su sociedad, han sido victimizadas por actos de violencia de género y que a veces tienen dificultad para reconocer estos actos como VG (Barredo et al., 2014).

Precisamente por esta cualidad insidiosa de los prejuicios y sesgos implícitos que se han interiorizado a través de procesos identitarios, es de particular importancia conocer los mecanismos cognitivos y comportamentales que mantienen la aceptación de la VG en docentes (Pérez \& Hernández, 2009). Es de igual valor explorar cómo estos sesgos y prejuicios se relacionan de manera directa con actitudes sexistas que puedan tener los docentes hacia su alumnado. Esto permitirá que los científicos generen datos que puedan formar programas de prevención que atiendan necesidades reales, iniciando por las barreras actitudinales, las cuales suelen cobrar particular importancia en el momento en que los sistemas de protección infantil dependen de estas detecciones como punto de entrada al sistema de protección (Piedra et al., 2014).
En el contexto de República Dominicana, el rol de la mujer ha experimentado pocas transformaciones trascendentales en los últimos años. El valor de la mujer dominicana aún está íntimamente atado a su capacidad reproductiva. De igual manera, la hiper-sexualidad conferida a adolescentes en la publicidad, medios de comunicación masiva y cultura general, mantiene a la sexualidad de la mujer en una posición subyugada al poder masculino. En este contexto, la sexualidad femenina es explotada exclusivamente para el placer de los hombres heterosexuales. Este abordaje coexiste con actitudes características del sexismo benevolente (Glick \& Fiske, 2001). El sexismo benevolente es un tipo de sexismo en el cual se valora la mujer exclusivamente por su capacidad cuidadora y reproductiva. En este imaginario la mujer es pasiva y débil, por lo que para desempeñar sus funciones requiere de la protección y el cuidado de los hombres. Esta representación de la mujer enfatiza su belleza, delicadeza y abnegación. Esto contrasta con el sexismo hostil, el cual visualiza a la mujer como aquella que desea poseer, dominar y controlar a los hombres. A la vez, atribuye características negativas a la sexualidad femenina (Glick \& Fiske, 2001). Si bien ambas representaciones de la mujer dominicana parecen discrepar, ambas tienen en común que la mujer es un objeto pasivo cuyo valor positivo o negativo es otorgado por los hombres en función de sus necesidades particulares.

En este trabajo tratamos la violencia de género como una serie continua de creencias y conductas que comparten como denominador común culpar a la mujer por su victimización y por su posición inferior en la jerarquía social. Esta culpa proviene de la creencia de que la mujer merece ser castigada cuando no acepta de manera pasiva su posición en la jerarquía social de la cultura predominante en la cual el hombre heterosexual ocupa la posición de mayor poder. Estas creencias lo que hacen es normalizar la masculinidad tradicional y sus consecuencias, entre ellas la violencia de género, la cual es utilizada como mecanismo de coerción y control (Expósito et al., 2014). Dada esta definición, si conceptualizamos la violencia de género como un sistema complejo de creencias y comportamientos organizados por procesos psico- 
lógicos y sociales de construcción de identidad, y no como acciones o sucesos discretos de desviación conductual, podemos comprender mejor cómo las personas manejan esta serie de actitudes y conductas. Este abordaje es importante porque desvincula a la violencia machista de la psicopatología particular.

Este esquema permitirá explorar si existen maneras de predecir las formas más violentas y agresivas de la violencia basada en el género, así como manifestaciones no violentas, pero igualmente discriminatorias de estas creencias en diversos contextos (Mingo, 2010; Pérez \& Hernández, 2009). En este sentido, adherencia a creencias de sexismo benevolente o simbólico pueden predecir conductas explícitamente violentas como la violación y el feminicidio. La literatura científica sugiere que este modelo es viable (McMahon \& Farmer, 2011).

Para fines de esta investigación, cuyo objetivo es explorar si las creencias sexistas de los docentes se relacionan con la percepción de las habilidades del alumnado, se realizó un análisis secundario de los datos de monitoreo y evaluación del proyecto Leer, en el cual se identificaron tres tipos de violencias de género: el acoso sexual laboral, la violencia entre parejas íntimas y la violación sexual (Sánchez-Vincitore et al., 2017). El proyecto seleccionó estos tres tipos de VG por su frecuencia en la cultura local y porque permite tomar mediciones de actitudes diversas como parte del término sombrilla de VG. Se omiten otros tipos, como violencia obstétrica, por estar restringida solo a mujeres que han tenido hijos. A la vez, se propone que estas actitudes se comportan como esquemas cognitivos, lo que hace que su impacto sea aún mayor en el comportamiento, ya que los esquemas cognitivos impactan la interpretación de eventos y guían las respuestas comportamentales ante los mismos.

Como se propone la VG como un grupo continuo de actitudes y comportamientos, hipotéticamente la violación sexual se podría estimar con base en creencias más sutiles como el acoso sexual laboral y la violencia doméstica. Entendemos que esto es posible dado que la deseabilidad social (o normas sociales) impediría la aceptación franca de la violación, mien- tras que formas más sutiles y aceptables — como el acoso laboral- permiten que las personas develen sus actitudes con cierta facilidad. Entendemos el acoso sexual laboral como la gama de conductas experimentadas por las mujeres en el entorno de su trabajo (formal o informal), caracterizadas por comentarios inapropiados sobre la apariencia física de las mujeres, la exigencia a mujeres afrodescendientes de conformarse a estándares de belleza eurocéntricos para ser consideradas profesionales, hasta relaciones sexuales coaccionadas a cambio de promociones y beneficios salariales (Expósito et al., 2014).

Conceptualizamos la violencia de pareja como aquella que se da en la dinámica de poder en las relaciones de parejas heterosexuales, en las cuales la violencia tiene un rango que va desde el control de la conducta de la mujer (control sobre amistades, actividades sociales, vestimenta, etc.), hasta celos, golpes y violación matrimonial. Las secuelas de estas formas de violencia son ampliamente conocidas, impactando negativamente la salud mental y la productividad laboral (Expósito et al., 2014). Para fines de este estudio no se explora la violencia entre parejas íntimas gais y lesbianas, ya que la legislación dominicana vigente no reconoce el matrimonio igualitario.

Este estudio también explora cómo las actitudes de aceptación de estos tres tipos de violencia hacia la mujer se relacionan con la práctica docente. Culturalmente, la conceptualización del docente y su rol en la escuela se ha centrado en las competencias que el mismo posee para impartir contenidos curriculares. Si bien desde el Estado existe el debido enfoque en asegurar la calidad docente a través de la medición de la competencia de estos, en raras ocasiones se toma en consideración cómo las creencias y la estructura de la personalidad del docente impacta su práctica en el aula.

Partiendo de este supuesto, es válido contemplar si los docentes de las escuelas, que son parte de la cultura dominicana y comparten los guiones de masculinidad de la cultura, están contribuyendo a la enseñanza del machismo y perpetuando la desigualdad y la violencia a través de su práctica docente. 
La literatura científica ha evidenciado que hasta las ansiedades de los docentes impactan la percepción que tienen los estudiantes sobre sus competencias, particularmente sus competencias para las matemáticas (Beilock et al., 2010).

\section{Método}

Los datos utilizados para este análisis secundario provienen del sistema de monitoreo y evaluación del proyecto Leer; una iniciativa de la Agencia de los Estados Unidos para el Desarrollo Internacional (USAID por sus siglas en inglés). El enfoque principal del proyecto es mejorar la lectura y escritura en 200,000 niños que cursan los primeros seis grados de educación primaria en escuelas públicas dominicanas. El proyecto tiene además un componente de calidad del ambiente escolar, a través del cual se trabaja la inclusión educativa de niños y niñas con discapacidad y necesidades específicas de apoyo educativo, la reducción del bullying o acoso escolar y la reducción de la aceptabilidad de la violencia de género por parte de los docentes y otros actores clave del sistema educativo. Los datos de este análisis provienen del levantamiento de datos que se realiza al final de cada año escolar (junio), con el fin de evaluar si las variables de interés del proyecto han mejorado en función de la intervención. Los datos de este análisis provienen del segundo año de implementación del proyecto en las escuelas, el cual culminó en junio del año 2017. El análisis secundario realiza unas funciones adicionales a las del monitoreo rutinario de indicadores del proyecto, buscando hacer inferencias importantes sobre cómo las actitudes impactan la práctica docente, enriqueciendo así el cambio de acción de proyectos educativos.

\subsection{Participantes}

Los participantes del estudio fueron 124 docentes y directores de escuelas públicas de diversas regionales educativas del sistema dominicano. La mayoría de los docentes impartían docencia en el segundo grado de primaria en las escuelas públicas que participaban en el proyecto Leer en el año 2017. Las escuelas fueron seleccionadas de manera aleatoria entre las escuelas que participan en el proyecto.

\subsection{Instrumentos}

Para levantar datos fiables se realizaron adaptaciones de escalas validadas que miden tipos de violencia de género. A la vez, se creó un cuestionario sociodemográfico que levantó datos sociodemográficos comunes en investigaciones (sexo, edad, nivel educativo), así como valoraciones relacionadas con roles o estereotipos de género. Esta sección del cuestionario sociodemográfico incluyó preguntas sobre quiénes son más emotivos, más aptos para las matemáticas y más cualificados para tareas domésticas, entre otras. Las escalas fueron traducidas por un proceso de traducción simultánea por dos expertos y luego se convocó un comité de expertos para consensuar el ítem final. Esto permitió evaluar que los ítems no se referían exclusivamente a traducciones literales y que cada reactivo funcionara en la cultura local. Este trabajo de adaptación y psicometría se realizó al iniciar el proyecto en el 2015 y se emplearon las mismas versiones de los instrumentos en esta medición (Mencía-Ripley et al., 2015).

\subsubsection{Escala de Illinois de Aceptación de Mitos de la Violación}

Se evaluó la aceptación de los mitos de la evaluación con el IRMA o la Escala Illinois de Aceptación de Mitos de la Violación. Dicho instrumento fue adaptado al espańol y al contexto cultural dominicano a través de un proceso de traducción paralela y, luego, por una valoración de expertos. Se seleccionó este instrumento originalmente por sus buenas propiedades métricas (McMahon \& Farmer, 2011). El formato de respuesta fue modificado a uno de cuatro puntos tipo Likert, en el cual uno indica total desacuerdo y cuatro total acuerdo. Ningún ítem fue codificado en reversa. Luego de la valoración grupal por expertos, la versión final fue probada en un estudio piloto con un grupo de docentes. Para este levantamiento de datos el coeficiente de consistencia interna fue $\alpha=.87$.

\subsubsection{Escala Illinois de Aceptación del Acoso Sexual}

Se utilizó la Escala Illinois de Aceptación del Acoso Sexual. La escala tiene propiedades métricas adecua- 
das e investigaciones han demostrado su uso apropiado en países hispanoparlantes (Expósito et al., 2014). El proceso de traducción y adaptación fue igual al antes descrito. En el primer uso masivo de la escala para el proyecto se obtuvo un coeficiente de consistencia interna de 90 .

\subsubsection{Escala de Aceptación de Mitos de la Violen- cia Doméstica}

Utilizamos este instrumento (Peters, 2008), el cual fue adaptado y traducido según los procedimientos anteriormente descritos. Al igual que las demás escalas ya descritas, el instrumento mostró buenas propiedades de consistencia interna.

El cuadernillo de preguntas incluyó también una serie de preguntas generadas para este estudio que indagan sobre roles y comportamientos basados en género y competencias académicas de los estudiantes. Sobre roles y comportamientos, las preguntas pedían a los docentes identificar si los niños o las niñas son más emotivos o sensibles, y si los niños y las niñas deben cuidar de otros y realizar tareas del hogar. Para las asignaturas académicas creamos una escala de respuesta para las competencias en Lectura, Matemáticas, Ciencias y Deportes, de cinco puntos tipo Likert. Mientras más alta la puntuación, mayor la creencia en que los niños son mejores que las niñas.

\subsection{Procedimiento}

Los docentes de segundo de primaria fueron invitados a participar en el estudio por los acompañantes del proyecto. Los acompañantes son miembros del equipo que implementa el proyecto Leer en las escuelas y su labor principal es realizar observaciones en aula y asistir a los docentes en la mejora de su práctica pedagógica. Las escuelas que participaron en el estudio fueron seleccionadas de manera aleatoria de las 400 escuelas que participaban en el proyecto. Los criterios de inclusión de las escuelas en el proyecto fueron: pertenencia al área geográfica urbana, presencia de un psicólogo u orientador en el plantel y programa de jornada extendida. Los datos de esta evaluación provienen de una evaluación anual que se realiza del proyecto al finalizar cada año escolar. Los docentes que accedieron a participar recibieron un documento de consentimiento informado, así como el cuadernillo con los cuestionarios y escalas antes descritos. Los docentes llenaron los cuestionarios de manera individual y, al completarlos, lo entregaron a los acompañantes, quienes los llevaron a la universidad para digitación, control de calidad y análisis. Las iniciativas de investigación del proyecto Leer cuentan con la aprobación el Comité de Ética de la institución que implementa el proyecto.

\section{Resultados}

\subsection{Descripción sociodemográfica}

El promedio de edad de los participantes fue de 43 años de edad $(N=124 ; S D=7.98)$. La mayoría de los docentes fueron mujeres $(85.7 \%)$ y solo un $27.1 \%$ de docentes contaba con una titulación de postgrado (especialidad, maestría o doctorado). La mayoría de los participantes estaban casados (56.4\%). El promedio de años de experiencia en el magisterio fue de 13 ańos al momento de completar los instrumentos $(D E=8.94)$. Para fines de estos análisis no se separarán los resultados por directores y docentes, pues muchos directores del sistema tienen funciones docentes en sus escuelas. A la vez, en vista de que muy pocos docentes que participaron eran hombres, se decidió tratar al grupo por su calidad docente y no desagregar por sexo biológico, pues las características antes descritas no permitirían muestras adecuadas para dicho contraste. En este estudio no levantamos datos sobre identificación étnica y otras variables, ya que trascienden el enfoque del proyecto y el estudio.

\subsection{Percepción de los docentes de los roles de género}

Los análisis realizados demostraron que la mayoría de los docentes no estuvieron de acuerdo con algunos aspectos de los roles de género tradicionales. Específicamente, solo un $22 \%$ de docentes creen que todas las mujeres aspiran a la maternidad y que los padres son los principales proveedores de la familia. No obstante, los docentes tenían otras percepciones altamente estereotipadas. En general, los y las docentes expresaron que las nińas son lloronas (70.6\%) 
y mucho más emotivas y sensibles que los niños (86.8\%). Más de la mitad de los docentes (58.8\%) aún cree que las niñas son más aptas para los trabajos de la casa que los niños. De igual forma, la mayoría opina que la crianza de los niños y niñas debe estar a cargo de las mujeres (64.7\%). Finalmente, la mayoría opinó que los niños son más fuertes (físicamente) que las niñas (82.4\%).

Sobre la aceptación de mitos de violencia, el promedio sugiere que hay aceptación de la violación $(M=$ 2.26, $D E=.44)$, acoso sexual $(M=2.39, D E=.41)$ y la violencia entre parejas íntimas $(M=2.46, D E$ $=.38$ ). Calculamos también la media para creencia sobre habilidades académicas y deporte que se incluyeron en el cuestionario sociodemográfico. Las puntuaciones sobre 2.5 muestran una tendencia a favorecer a los varones. Encontramos que las medias para matemáticas $(M=3.28, D E=.75)$ y para los deportes $(M=3.68, D E=.65)$ favorecen la creencia que los niños son mejores que las niñas en estas actividades académicas.

\subsection{Estadísticas inferenciales}

Como argumentamos que todas las formas de violencia de género existen en un continuo de violencia y que los tipos de violencia no corresponden a distintas variables o variables discretas, esperamos que las tres formas o manifestaciones de la violencia se relacionaran. Se realizó un análisis de correlación para establecer la relación entre las tres dimensiones de violencia seleccionadas para este estudio. Los resultados demuestran que todas las correlaciones fueron significativas $(p<.01$, dos colas).

Exploramos también si la edad de los docentes se relacionaba con la aceptación de la violencia. Esperábamos específicamente que docentes más jóvenes, quienes han vivido momentos culturales donde los roles de género han cambiado un poco y han tenido la experiencia de las iniciativas de igualdad de género de las políticas públicas más recientes, expresaran menor aceptación de la violencia de género. No obstante, la edad no estuvo significativamente correlacionada con ninguna de las variables de violencia de género.
Finalmente, realizamos un análisis de regresión para explorar cuánto predicen la aceptación de mitos de violencia (variables independientes) en sus tres variantes (violación, violencia entre parejas íntimas y acoso sexual laboral) las creencias de los docentes sobre las competencias y aptitudes de los estudiantes para Lengua Española, Matemáticas, Ciencias y Deportes (variables dependientes). El único modelo estadísticamente significativo fue el de Matemáticas $(F(4920,3)=2.82, p<.05)$ y para el cual se explica el $12 \%$ de la varianza $\left(R^{2}=.118\right)$.

Cuando observamos cada variable en el modelo, vemos que la violencia entre parejas íntimas no fue significativa, pero que la violación sexual $(t=2.52$, $p=.014)$ y el acoso sexual fueron significativos $(t$ $=-2.36, p=.022)$. Realizamos también diagnósticos de colinealidad y los valores relacionados con el factor de inflación de varianza (inferiores a 4), así como los bajos indicadores de tolerancia (inferiores a $.4)$, indican que si bien las variables están altamente correlacionadas, las mismas se refieren a constructos distintos. En este sentido, se cumplen los supuestos necesarios para realizar este análisis.

\section{Conclusiones}

Este estudio exploró cómo tres variables que forman parte de la violencia de género (violación, acoso sexual laboral y violencia entre parejas íntimas) se relacionan con las creencias de los docentes sobre las competencias académicas de sus estudiantes de primaria. Los participantes fueron mayormente mujeres. Los resultados mostraron que el modelo fue estadísticamente significativo para las Matemáticas, indicando que la aceptación de la violencia de género solo se relaciona con las creencias de los docentes en torno a aptitudes y habilidades matemáticas de los alumnos. Esto no ocurre para las otras asignaturas académicas exploradas en este estudio (Deportes, Ciencias y Lenguas). Específicamente, la aceptación de mitos sobre la violación sexual se asocia a la mayor percepción de competencias matemáticas por parte de los varones. La relación negativa para acoso sexual implica que mientras los docentes aceptan más los mitos del acoso sexual, 
creen que los niños son menos competentes en matemáticas que las niñas.

Estos hallazgos sugieren que los docentes que aceptan la masculinidad tradicional (p. ej., agresión, poder, control) no solo aceptan mitos sexistas sobre la violación sexual, sino que también atribuyen mayores capacidades a los niños para las matemáticas. Estos datos son congruentes con otras investigaciones de la región en los cuales los docentes tienen las creencias de que las mujeres provocan las violaciones y otros actos de VG (Barredo et al., 2014). Por otro lado, cuando estas personas también aceptan el acoso sexual laboral, la tendencia es la opuesta. Esto puede ser porque los mitos de acoso sexual suelen proyectar a las mujeres como manipuladoras o engañosas (sexismo hostil), por lo cual en este caso el esquema de poder paradójicamente favorece a las mujeres. Aunque el poder atribuido a las mujeres en este caso es negativo.

El constructo subyacente que puede ayudar a entender estos hallazgos es el sexismo hostil. El sexismo hostil es el que define la masculinidad como poderosa, controladora y agresiva. Pero a diferencia del sexismo benevolente, en el cual la mujer es vista como frágil y delicada, lo cual la hace necesitar la protección y el cuidado del hombre, el sexismo hostil caracteriza a la mujer de manera negativa. En el sexismo hostil la mujer no es vista como un sujeto pasivo, sino como una persona manipuladora y seductora cuyo móvil es dominar y controlar a los hombres (Glick \& Fiske, 2001). Esta interpretación se ve fortalecida por el análisis de colinealidad, el cual demostró que las tres variables están altamente correlacionadas, pero que no hay multicolinealidad, sugiriendo que hay un constructo o variable latente que puede ser la matriz de la cual provienen estas tres actitudes, las cuales son similares, pero a la vez se diferencian en su manifestación comportamental.

Finalmente, debemos tener en cuenta que la mayoría de los participantes de esta investigación fueron mujeres. Esto nos indica que estas creencias consistentes con el sexismo hostil parten de mujeres, por lo que podemos estar visualizando la adopción o la acepta- ción del sexismo por parte de las mujeres. Las implicaciones de la aceptación del sexismo hostil para las mujeres es un área que amerita mayor estudio, ya que la identidad se relaciona con diversos aspectos del funcionamiento humano, tales como la salud mental y el logro académico. Numerosos estudios han establecido esto para la identidad étnica, y es posible que la identidad de género tenga efectos similares (Roberts et al., 1999; Smith \& Silva, 2011).

En función de estos hallazgos, las intervenciones de igualdad de género no pueden descansar en que solo se instauren nuevas políticas y nuevos procedimientos administrativos en las escuelas, ya que los mismos no trabajan explícitamente las creencias o cogniciones que tienen los docentes en torno a actitudes relacionadas con el sexismo hostil. Estas actitudes han sido modeladas en el hogar, la escuela y la vida pública de los docentes; de hecho, investigaciones sugieren que la aceptación de la violencia de género se comporta como un esquema cognitivo (Süssenbach et al., 2015). De igual manera, si las mujeres han interiorizado aspectos del sexismo hostil, sólo incrementar la participación de las mujeres en el entorno laboral y en la toma de decisiones no llevará a los cambios actitudinales necesarios para promover la igualdad de género. Esto es de particular importancia en el ámbito del magisterio, ya que a pesar de que la profesión está altamente feminizada, los puestos administrativos y de mayor jerarquía en los sistemas educativos siguen estando ocupados principalmente por hombres y su ocupación por parte de mujeres no garantiza mayor equidad si las mismas forman parte de la continuación de la desigualdad a través de la participación en discursos machistas (Barquín \& Melero, 1994).

El bajo impacto de políticas de género ya se ha medido (Henry, 2015), especialmente en las ciencias, por lo que urgen estudios que contextualicen el sexismo hostil en América Latina para proponer acciones viables al respecto. Este último aspecto cobra importancia particular por la evidencia existente sobre cómo las actitudes de los docentes impactan los logros académicos de sus estudiantes y cómo la ansiedad que sienten las niñas en torno a las matemáticas 
impactan negativamente su desempeño (Van Mier, Schleepen \& Van den Berg, 2019).

\section{Agradecimientos}

La base de datos analizada para este estudio proviene del proyecto Leer. Ello fue posible gracias al generoso apoyo del pueblo americano a través de la Agencia de los Estados Unidos para el Desarrollo Internacional (USAID). Los contenidos son responsabilidad del autor y no reflejan los puntos de vista de USAID o el gobierno de los Estados Unidos. El autor agradece a la Dra. Laura Sánchez-Vincitore y al Prof. Carlos Ruiz Matuk.

\section{Referencias}

Artal, M. (2009). Construir el género. El cuestionamiento del sexismo y del androcentrismo en el sistema educativo. Acciones e investigaciones sociales, (27), $5-21$.

Barquín Ruiz, J., \& Melero Zabal, M. (1994). Feminización y profesión docente: Internalización sexista del trabajo. Revista de Investigación en la Escuela, 22, 25-34.

Barredo Ibáñez, D., Liberia Vayá, I., Macías Cruzatty, A., Palomeque Guillén, V., Delgado Burgos, M. Á., Zurbano-Berenguer, B., Espinoza-Lucas, M., García García, M. E., \& Cedeño Delgado, G. (2014). Actitudes y percepciones sobre la violencia de género del personal docente y administrativo y de servicios de Manta: estudio del Colegio 5 de Junio (2014). Revista San Gregorio, 1, 38-45. https://bit.ly/32urG4f

Beilock, S. L., Gunderson, E. A., Ramirez, G., \& Levine, S. C. (2010). Female teachers' math anxiety affects girls' math achievement. Proceedings of the National Academy of Sciences, 107(5), 1860-1863. https://doi.org/10.1073/pnas.0910967107

Carli, L. L., Alawa, L., Lee, Y., Zhao, B., \& Kim, E. (2016). Stereotypes about gender and science: Women $\neq$ scientists. Psychology of Women Quarterly, 40(2), 244-260. https://doi.org/10.1177/0361684315622645

Eagly, A.H., \& Miller, D.I. (2016). Scientific Eminence: Where are the women? Perspectives on Psychological Science, 11(6), 899-904. https://doi.org/10.1177/1745691616663918

Friedrich, A., Flunger, B., Nagengast, B., Jonkmann, K., \& Trautwein, U. (2015). Pygmalion effects in the classroom: Teacher expectancy effects on students' math achievement. Contemporary Educational Psychology, 41, 1-12.

https://doi.org/10.1016/j.cedpsych.2014.10.006

Expósito, F., Herrera, A., Valor-Segura, I., Herrera, M. C., \& Lozano, L. M. (2014). Spanish adaptation of the Illinois sexual harassment myth acceptance. The Spanish Journal of Psychology, 17.

https://doi.org/10.1017/sjp.2014.42

Glick, P., \& Fiske, S. T. (2001). An ambivalent alliance: Hostile and benevolent sexism as complementary justifications for gender inequality. American psychologist, 56(2), 109.

https://doi.org/10.1037/0003-066X.56.2.109

Heise, L., Ellsberg, M., \& Gottmoeller, M. (2002). A global overview of gender based violence. International Journal of Gynecology \& Obstetrics, 78, S5-S14. https://doi.org/10.1016/S0020-7292(02)00038-3

Henry, F. (2015). Survey of Women in the Academies of the Americas. Interamerican Network of Academies of Sciences. https://bit.ly/32A1y8p

López Ojeda, E. (2006). Análisis sociolingüístico: pautas de observación y análisis del sexismo. Los materiales educativos. Interlingüistica, (17), 630-639. https://bit.ly/2R9C2V3

McMahon, S., \& Farmer, G. L. (2011). An updated measure for assessing subtle rape myths. Social Work Research, 35(2), 71-81. https://doi.org/10.1093/swr/35.2.71

Mencía-Ripley, A., Sánchez-Vincitore, G., Garrido, L., \& Aguasvivas, J. (2015). USAID-Leer Baseline Report. https://bit.ly/2NMXhsw 
Mingo, A. (2010). Ojos que no ven...Violencia escolar y género. Perfiles Educativos, 32(130), 25-48.

National Sexual Violence Resource Center (NSVRC) (2015). Statistics about Sexual Violence. http://bit.ly/2kVs2kE

Pérez Martínez, V. T., \& Hernández Marín, Y. (2009). La violencia psicológica de género, una forma encubierta de agresión. Revista Cubana de Medicina General Integral, 25(2).

Peters, J. (2008). Measuring myths about domestic violence: Development and initial validation of the domestic violence myth acceptance scale. Journal of Aggression, Maltreatment \& Trauma, 16(1), 1-21. https://doi.org/10.1080/10926770801917780

Piedra, J., García Pérez, R., Fernández García, E., \& Rebollo, M.A. (2014). Brecha de género en educación física: Actitudes del profesorado hacia la igualdad. Revista Internacional de Medicina y Ciencias de la Actividad Física y el Deporte, 14 (53), 1-21. https://bit.ly/2WV9xLK

Plan Internacional República Dominicana (2017). Niñas Esposadas. Caracterización del matrimonio infantil forzado en las provincias de Azua, Barahona, Pedernales, Elías Piña y San Juan. Planteamientos (2), 1-32. https://bit.ly/33xcvIS

Roberts, R. E., Phinney, J. S., Masse, L. C., Chen, Y. R., Roberts, C. R., \& Romero, A. (1999). The structure of ethnic identity of young adolescents from diverse ethnocultural groups. The Journal of Early Adolescence, 19(3), 301-322.
https://doi.org/10.1177\%2F0272431699019003001

Sánchez-Díaz, L. Street harassment perception and it relations with self-objectification of women. INTERdisciplina, 7(17), 153-170.

https://doi.org/10.22201/ceiich.24485705e.2019.17.67534

Sánchez-Vincitore, L.V., Garrido, L.E., Martínez, L., \& Mencía-Ripley, A. (2017). End of Second Grade Reading Assessment of USAID's Read Project: Academic Year 2016-2017. https://bit.ly/2CtjQgR

Smith, T. B., \& Silva, L. (2011). Ethnic identity and personal well-being of people of color: A meta-analysis. Journal of Counseling Psychology, 58(1), 42. https://doi.org/10.1037/a0021528

Süssenbach, P., Eyssel, F., Rees, J., \& Bohner, G. (2017). Looking for blame: Rape myth acceptance and attention to victim and perpetrator. Journal of interpersonal violence, 32(15), 2323-2344. https://doi.org/10.3389/fpsyg.2018.02690

Süssenbach, P., Eyssel, F., Rees, J., \& Bohner, G. (2017). Looking for blame: Rape myth acceptance and attention to victim and perpetrator. Journal of interpersonal violence, 32(15), 2323-2344. https://doi.org/10.1177\%2F0886260515591975

Van Mier, H. I., Schleepen, T. M. J., \& Van den Berg, F. C. G. (2018). Gender Differences Regarding the Impact of Math Anxiety on Arithmetic Performance in Second and Fourth Graders. Frontiers in Psychology, 9, 2690. https://doi.org/10.3389/ fpsyg.2018.02690

\section{CÓMO CITAR:}

Mencía-Ripley, A. (2020). Aceptación de la violencia de género en docentes de escuelas públicas. Revista Caribeña de Investigación Educativa (RECIE), 4(1), 81-91. https://doi.org/10.32541/recie.2020.v4i1.pp81-91 